\title{
Effect of Prior etching on Bond Strength of Self-Etch adhesive in case of Sensitive Teeth treated with CPP-ACP.
}

\author{
Suruchi Chaudhary Tayal ${ }^{1}$ \\ Jaidev Dhillon ${ }^{2}$
}

Abhishek Tayal ${ }^{3}$

\section{ABSTRACT}

Introduction: The management of dentinal hypersensitivity involves remineralization by various means, one of which being CPP-ACP paste, followed by restoration. It was speculated that the bond strength of self-etching adhesive was affected following the application of CPP-ACP paste and prior etching with phosphoric acid.

Aims and objectives: To evaluate the tensile bond strength (TBS) of G-Bond (GB, self-etching adhesive) to dentin following the application of CPP-ACP (Tooth Mousse/TM), with and without prior etching.

Materials and method: Freshly extracted forty five permanent molars were collected for the study. All the teeth were ground and polished to expose the flat dentinal surface. The teeth were embedded in the resin in separate molds and were divided into three equal groups of fifteen samples each Group 1: Samples treated with Tooth Mousse for 60 min daily for 7 days and then restored with GB and composite (Filtek Z350, 3M ESPE). Group 2: Samples treated with Tooth Mousse and etched with phosphoric acid prior to restoration with GB and composite. Group3: Samples restored with GB and composite. A split brass mold was placed over the predesigned location and with help of the plasma coated instrument, composite resin was placed. Tensile loading was done using Universal Testing Machine.

Conclusion: TBS of GB was not significantly reduced after CPP-ACP application, but after prior etching the CPP-ACP specimens, the bond strength of GB was significantly reduced.

Keywords: dentin hypersensitivity, self-etch adhesive, $C P P-A C P$.

\section{INTRODUCTION}

Dentinal hypersensitivity is a significant clinical problem, in which a sound, exposed dentinal surface is sensitive to stimuli that would normally cause no discomfort. ${ }^{1}$ It is defined as a short and sharp pain arising from exposed dentin, in response to chemical, thermal, tactile or osmotic stimuli, that cannot be explained as arising from other forms of old dental defect or pathology. ${ }^{2}$ Dentin may become exposed due to wear, caries and the development of noncarious cervical lesions. ${ }^{3}$ Non-carious cervical lesions are defined as the loss of tooth substance at the cemento-enamel junction. Terms which are used to describe these are 'cervical erosion/abrasion' lesions and 'abfractions'. The term 'abfraction' evolved from the work by McCoy (1982), Lee and Eakle (1984), and Grippo (1991). ${ }^{4}$ When cervical dentin is exposed, either from loss of overlying enamel or from loss of gingiva covering the root, tooth structure loss may accelerate unless etiological factors are determined and eliminated. Thus, exposed dentin in the cervical area, with or without the loss of tooth structure, may become sensitive. The difficulty found in treating dentin hypersensitivity is expressed by the enormous number of techniques and therapeutic alternatives to relieve it. Several materials such as varnishes, liners, restorative materials, dentinal adhesives $^{5}$, dentifrices and mouthwashes are used to reduce dental sensitivity. ${ }^{6}$
Casein phosphopeptide-amorphous calcium phosphate (CPP-ACP) is ACP that is complexed with the milk protein, casein phosphopeptide. Casein phospho-peptides (CPP) containing the cluster sequence [-Ser(P)-Ser(P)-Ser(P)-GluGlu] have a remarkable ability to stabilize calcium phosphate in solution and substantially increase the level of calcium phosphate in dental plaque. ${ }^{7}$ It helps to suppress the demineralization and promote mineralization of enamel by deposition of apatite. The casein phosphopeptide (CPP) contains phosphoseryl sequences which get attached and stabilized with amorphous calcium phosphate (ACP). ${ }^{8}$ The effects of casein phospho-peptides (CPP) in reducing demineralization and enhancing remineralization in tooth enamel have been investigated by measuring the effect of CPP-ACP on calcium diffusion in plaque ${ }^{9}$ and in laboratory and in situ studies CPP-ACP has shown to remineralize human enamel subsurface lesions..$^{10,11,12}$ Furthermore, remineralized enamel has shown to be more resistant to subsequent acid attack. Therefore, CPP-ACP has been incorporated into oral health products, such as chewing gums and mouthwashes. It has been incorporated into commercial products such as:

1. GC Tooth Mousse ${ }^{\mathrm{TM}}$ (water based, sugar free crème with various flavors available).

2. GC MI Paste Plus ${ }^{\mathrm{TM}}$ (with the incorporation of $900 \mathrm{ppm}$ fluoride).

1. Senior Lecturer, 2. Professor and Head, Department of Conservative Dentistry and Endodontics, B.R.S Dental College and Hospital.

3. Senior Lecturer, Department of Prosthodontics, B.R.S Dental College and Hospital. 
3. Recaldent ${ }^{\mathrm{TM}}$ Chewing Gum (xylitol and CPP-ACP).

4. Recaldent ${ }^{\mathrm{TM}}$ Lozenges.

Self-etching primer adhesives present the dentist with the application of fewer application steps, and therefore lesser technique sensitivity, reduced operative time, and have been shown to form reliable bonds to enamel as well as dentine. The self-etching adhesives are the acidic primers and the rationale behind these systems is to superficially demineralise dentin and to simultaneously penetrate it to the depth of demineralization with monomers that can be polymerized in situ. ${ }^{13}$ Recent studies have shown that, use of phosphoric acid etching with the self-etching primer adhesives significantly reduces the bond strength to dentin. ${ }^{14}$

The dentin smear layer is composed of small particles of mineralized collagen matrix spread over the dentinal surface, inorganic tooth particles, saliva, blood and numerous microorganisms. ${ }^{15}$ This smear layer seals the opening of dentinal tubules and covers the intertubular and peritubular dentin. It was speculated that bond strength of adhesive and etching of CPP-ACP treated samples before applying self-etching adhesive also does not effect the bond strength.

METHOD: Forty five intact caries free mandibular permanent molars were obtained from the Department of Oral and Maxillofacial Surgery at D.A.V Dental College and Hospital, Yamunanagar. After the extraction, the teeth were cleaned of debris and blood in the running water. The calculus was removed with the help of scaler followed by cleaning with pumice slurry and rubber prophylaxis cup rotating at a slow speed in the contrangle micromotor handpiece (Unicorn, NSK). Each stored tooth was thoroughly washed with water and air dried. The teeth were stored in $1 \%$ chloramine $\mathrm{T}$ for two weeks and transferred to phosphate buffered saline solution During sample preparation, to standardize the depth of cavity on the occlusal surface, depth holes $1.5 \mathrm{~mm}$ were drilled in the deepest part of the central fossa of each tooth with the help of round diamond bur (SF 21, Prime and Dental, Mumbai). Uniform depth holes were drilled just to reach upto the dentin. All the teeth were ground on the orthodontic trimmer

\section{MATERIALS AND METHOD}

MATERIALS: The materials used in the study were as follows:

\begin{tabular}{|l|l|c|l|}
\hline Material & Contents & Code & Manufacturer \\
\hline $\begin{array}{l}\text { SCOTCHBOND } \\
\text { MULTIPURPOSE } \\
\text { ETCHANT }\end{array}$ & $30-40 \%$ Phosphoric acid, water, amorphous, silica. & PA & $\begin{array}{l}\text { 3M ESPE, } \\
\text { St Paul, MN, USA }\end{array}$ \\
\hline G-BOND & $\begin{array}{l}\text { 4-Methacryloxyethyltrimellitate(4-META), urethane } \\
\text { dimetacrylate, triethyleneglycol dimethacrylate acetone, } \\
\text { distilled water. }\end{array}$ & GB & $\begin{array}{l}\text { GC Corp, } \\
\text { Tokyo, Japan }\end{array}$ \\
\hline TOOTH MOUSSE & $\begin{array}{l}\text { 5-10\% casein phosphopeptide amorphous calcium } \\
\text { phosphate, glycerol, pure water, zinc oxide, xylitol, } \\
\text { silicon dioxide, sodium carboxyl methylcellulose, guar } \\
\text { gum phosphoric acid, sodium saccharin, magnesium } \\
\text { oxide propylene glycol, ethyl-p hydroxybenzoate, } \\
\text { butyl-p hydroxybenzoate }\end{array}$ & TM & $\begin{array}{l}\text { GC Corp, } \\
\text { Tokyo, Japan }\end{array}$ \\
\hline
\end{tabular}

self-etching primer may be reduced on the changed dentinal surface when CPP-ACP is applied. Also, the use of etchants prior to the application of self- etching adhesive may affect the bond strength with dentin, as it improves the bond strength with enamel as reported in earlier studies. ${ }^{14}$

Thus, the aim of this study was

1. To determine whether the application of tooth mousse or CPP-ACP affects the tensile bond strength of selfetching adhesive.

2. To evaluate the effect of prior etching on self-etching adhesives in case of CPP-ACP treated teeth.

The null hypothesis was that pretreatment with CPP-ACP paste has no effect on the bond strength of self-etching until the drilled hole depth to expose the flat dentinal surface. This was followed by polishing of the flat dentinal surfaces with \#600 grit abrasive silicon carbide paper under copious running water. The teeth were then placed on the glass slab with the flat dentinal surfaces facing the glass slab and stabilized at the periphery with the wax sheet. Rectangular aluminium molds were placed in the position over the wax sheet bearing the teeth. A thin mix of autopolymerizing acrylic resin was placed in the molds to embed these teeth and the teeth were then stored in the normal saline. A punch hole tape with the diameter of $3 \mathrm{~mm}$ was placed over the occlusal surface.

The moulds were then randomly divided into three main groups of fifteen samples each. 
Group 1: Samples were treated with Tooth Mousse (GC Corp, Tokyo, Japan) for 60 min daily for 7 days. TM (CPPACP) was applied to fifteen samples by using an applicator brush to just cover the dentinal surface. The samples were then placed in the incubator with $95 \%$ relative humidity at 37 . water spray blast for 15 seconds and this procedure was repeated for 7 consecutive days. The samples were placed in the incubator throughout the study and were removed only for TM application and rinsing procedures. After application of Tooth Mousse, G-Bond (GC Corp, Tokyo, Japan) was applied according to manufacturer's instructions. No prior etching was done. Restoration was done with composite resin (Filtek Z350, 3M ESPE)

Group 2: Samples were treated with Tooth Mousse (GC Corp, Tokyo, Japan) for 60 min daily for 7 days. TM (CPPACP) was applied to fifteen samples as stated above. After application of CPP-ACP, dentin was etched with Scotchbond Multipurpose etchant (PA) for 15 seconds, gently rinsed for 10 seconds with an air water spray and the excess moisture was removed with air. Further, G-Bond (GC Corp, Tokyo, Japan) was applied according to manufacturer's instructions and restoration was done with composite resin (Filtek Z350, 3M ESPE).

Group 3: Samples were restored with G-Bond (GC Corp, Tokyo, Japan) and composite resin (Filtek Z-350, 3M ESPE): Using an applicator tip, a G-Bond (GC Corp, Tokyo, Japan) was applied on the dentinal surface, left undisturbed for 10 seconds and dried with a strong blast of air for 5 seconds as stated by manufacturer's instructions.

The bonded specimens were placed in the distilled water and kept in the incubator with $95 \%$ relative humidity at $37^{\circ} \mathrm{C}$. A split brass mould was placed over the bonded surface at the predesigned location. The bonding was done on a small circular area of $3 \mathrm{~mm}$ diameter as there is an inverse logarithmic relationship between the bond strength and the surface area for bonding. ${ }^{16}$ With the help of the plasma coated instrument, composite resin was placed incrementally in a cylindrical shape of $5 \mathrm{~mm}$ height and $3 \mathrm{~mm}$ diameter. The composite resin was placed in three equal increments which were cured for 20 seconds each. After the first increment of composite resin was placed, stainless steel wire loop was positioned so that it was perpendicular to the tooth surface. Twenty four hours after bonding, the specimens were removed from the incubator and tested in tensile mode in Universal Testing Machine (Llyod's Machine, CIPET, Amritsar). The equipment was adjusted to operate at $1 \mathrm{~mm} / \mathrm{min}$, as different cross head speeds may influence the bond strength values. For the tensile bond strength measurement the wire protruding out of the composite cylinder was gripped into the superior cross head and the aluminium mould was held in the inferior cross head of the Universal testing machine. Tensile loading was done until the dislodgement of the composite cylinder from the dentinal surface occurred. The breaking load was measured according to the readings on the display of the machine. The results of the tensile bond strength testing were tabulated in the value of force (Newton, $\mathrm{N}$ ) and were later converted into Mpa by dividing the load with the surface area of the specimen.

\section{RESULTS:}

The mean tensile bond strengths of various groups was as follows. Group-1 showed TBS of 69.43 Mpa, Group-2 showed TBS of 51.93 Mpa, Group-3 showed TBS of 71.4 Mpa .The mean difference in the bond strengths and standard error is shown in table 1.

Table 1:Comparison of three groups based on statististical analysis tests

\begin{tabular}{|c|c|c|c|c|c|c|}
\hline \multirow{2}{*}{ (I) group } & \multirow[b]{2}{*}{ (J) group } & \multirow{2}{*}{$\begin{array}{l}\text { Mean Difference } \\
\text { (I-J) }\end{array}$} & \multirow[b]{2}{*}{ Std. Error } & \multirow[b]{2}{*}{ Sig. } & \multicolumn{2}{|c|}{ 95\% Confidence Interval } \\
\hline & & & & & $\begin{array}{l}\text { Lower } \\
\text { Bound }\end{array}$ & $\begin{array}{l}\text { Upper } \\
\text { Bound }\end{array}$ \\
\hline \multirow{2}{*}{ GROUP 2} & GROUP 1 & $-17.50(*)$ & 2.29 & $.001 * *$ & -23.38 & -11.61 \\
\hline & GROUP 3 & $-19.47(*)$ & 1.98 & $.001 * *$ & -24.56 & -14.37 \\
\hline \multirow{2}{*}{ GROUP 1} & GROUP 2 & $17.50(*)$ & 2.29 & $.001 * *$ & 11.61 & 23.38 \\
\hline & GROUP 3 & -1.97 & 1.98 & .591 & -7.06 & 3.12 \\
\hline \multirow{2}{*}{ GROUP3 } & GROUP 2 & $19.47(*)$ & 1.98 & $.001 * *$ & 14.37 & 24.56 \\
\hline & GROUP 1 & 1.97 & 1.98 & .591 & -3.12 & 7.06 \\
\hline
\end{tabular}

* The mean difference is significant at the .05 level, highly significant at 0.001 level. 
The results clearly show that there was statistically insignificant difference $(p>0.05)$ between the tensile bond strengths of the Group-1 and Group-3. But, there was statistically significant difference $(p<0.001)$ between the tensile bond strengths of Group-2 and Group-3. The results were based on Tukey's HSD Test.

\section{DISCUSSION}

In the field of restorative dentistry dentinal hypersensitivity is one of the major challenges. Several theories have been proposed to explain the mechanism of dentinal hypersensitivity and the most accepted being the hydrodynamic theory proposed by Brannstrom. ${ }^{17}$ The elimination of the painful symptoms occurring during the dentinal hypersensitivity are done by the reduction of the fluid movement into the dentinal canalicules, through the occlusion of dentinal tubules.In a review article by Bartold PM, about dentinal hypersensitivity, he summarized the treatment strategies into a variety of self care as well as professional treatment strategies designed to depolarize the nerve or occlude and or seal the dentinal tubules. ${ }^{17}$ Management of hypersensitive dentin also involves remineralization or the occlusion of patent dentinal tubules and the agents used include fluoride containing solutions, oxalates and nitrates of potassium, potassium chloride or strontium chloride and amorphous calcium phosphates. However, the most common form of management is the placement of a topically applied agent either by a dental professional or by the patient at home.

The newest products aimed at hypersensitivity management are those containing amorphous calcium phosphate as the active ingredient. ACP forms hydroxyapatite in enamel and increases enamel hardness, hence its primary application as an agent is to remineralize carious lesion as well as reduce susceptibility to their formation. Products containing amorphous calcium phosphate also show promise for management of sensitivity via topical application. ${ }^{18,19}$ The topical form of calcium phosphate includes the application of CPP-ACP in form of tooth mousse. Tooth Mousse composition in detail includes CPP-ACP $10 \%$, Ca content $13 \mathrm{mg} / \mathrm{g}$, P content $5.6 \mathrm{mg} / \mathrm{g}$, and a $\mathrm{pH}$ of 7.8. The role of the CPP-ACP has been described as localization of ACP at the tooth surface which buffers free calcium and phosphate ion activities. The stabilized CPP-ACP prevents the dissolution of calcium and phosphate ions and maintains a supersaturated solution of bioavailable calcium and phosphates. Thus, this helps to maintain a super saturation with respect to the enamel which depresses the demineralization and enhances remineralization. ${ }^{20}$ Moreover, it has been reported that teeth treated with CPP-ACP are more resistant to acid attacks and decalcification and the treatment increases tooth remineralization as well. ${ }^{21}$

During the evolution of dentin bonding agents, attempts were made to ease out the technique and achieve good bonding with enamel and dentin under difficult oral conditions. In this process the recent development is combining etchant, primer and bonding agent in one component to form "self-etch adhesives". ${ }^{22}$ These selfetching adhesives can be used to etch both ground enamel and dentin simultaneously and they bond equally well to superficial and deep dentin. Self-etching primer adhesives bond micromechanically and chemically to dentine. These primers use acidic resin monomers to simultaneously etch and infiltrate the tooth surface. Thus, the success of the dentin bonding has been believed to be dependent on the infiltration of monomers into the acid etched dentin followed by the polymerization in situ. The hydrophilic monomers may form a complex structure with the exposed collagen fibers and the partially demineralised dentin containing the residual hydroxyapatite. G-Bond is an advanced 7th generation single component system which includes phosphoric ester monomer, 4-MET monomer, nanofilled particles. This "nano" level reaction produces insoluble compound for a better bond that is less likely to deteriorate from oral enzymes. It has simple application procedure and forms consistent and reliable bonds with enamel and dentin. $^{23}$

In this study, extracted teeth were cleaned off debris. The teeth were stored in $1 \%$ chloramine $\mathrm{T}$ for two weeks and transferred to phosphate buffered saline solution to avoid alteration in mechanical properties of dentinal surfaces. Uniform depth holes were drilled just to reach upto dentin as significantly lower bond strengths were observed in earlier done studies when dentin bonding agents were bonded to deeper dentin. ${ }^{24,25}$ After the flat dentinal surface was exposed, the polishing of the flat dentinal surfaces was done with \#600 grit abrasive silicon carbide paper under copious running water to produce a uniform smear layer, as it was reported that the smear layer created by diamond or steel bur was coarser, and its mechanical properties were weaker than that created by the $\# 600$ grit abrasive paper. ${ }^{26}$ The samples were divided into three equal groups as methodology was performed as mentioned earlier.

After obtaining the results, when Group 1 was compared to Group 3, slightly lower tensile bond strength of GB was observed after the application of CPP-ACP. This slightly lower bond strength could have been due to reduced etching efficiency of the adhesive after the application of the paste or 
due to the paste residues that impeded the adhesion. However, this decrease in bond strength was not statistically significant.

However, in Group 2, where the dentinal surfaces treated with CPP-ACP were further etched with phosphoric acid a significant decrease in bond strength was observed for self-etching bonding adhesive as compared to Group 1 . Several authors have suggested that phosphoric acid etching of the dentin opens up the dentinal tubules and exposes the collagen fibrils. After washing there is also a possibility of over-drying the etched dentin that leads to the collapse of the exposed collagen and incomplete infiltration into the areas which may compromise the bond strength. Any collapse even partial may hinder efficient resin infiltration leading to the porous zone at the bottom of the hybrid layer. ${ }^{27}$ According to Pashley et al, if the incomplete resin infiltration leaves the basal part of the hybrid layer without sufficient resin to completely envelope the demineralised collagen fibrils, it may leave the zone weaker than it should be and thus, making it susceptible to hydrolysis and microleakage. ${ }^{28}$

Van Meerbeck et al proposed that the phosphoric acid etching generally results in a rather abrupt transition of the exposed to the unaffected dentin so that the presently observed partially demineralised zone at the bottom of the hybrid layer must be ascribed to additional demineralization induced by the self-etching monomers. ${ }^{29}$ While the primer may have penetrated adequately the resin may still have been hampered to penetrate the exposed collagen network completely. Furthermore, Van Landuyt et al, reported that prior acid etching significantly increased the bonding effectiveness of the self-etching adhesive, Clearfil SE Bond, to enamel, however, doing likewise significantly decreased the bond strength to dentin. They speculated that the lack of a chemical reaction between MDP in the adhesive and hydroxyapatite resulted in lower bond strength, since hydroxyapatite is no longer available throughout the hybrid layer after acid etching. ${ }^{30}$

\section{CONCLUSION}

Under the limitations of this experimental study, the following conclusions were drawn:

1. The tensile bond strength of GB was not significantly reduced after the samples were treated with $\mathrm{CPP}-\mathrm{ACP}$ application for seven days and then restored with GB and composite resin.

2. Whereas, when the CPP-ACP treated samples were prior etched with phosphoric acid and later restored with $\mathrm{GB}$ and composite resin, the tensile bond strength of GB was significantly reduced.Thus, the null hyopothesis was rejected

\section{REFERENCES}

1. Ling TYY, Gillam DG. The effectiveness of desensitizing agents management. Br Dent J 1999; 187:606-611.

2. Addy M, Urquhart E. Dentine hypersensitivity: its prevalence, aetiology and clinical management. Dent Update. 1992; 19(10):4078, 410-2.

3. Walters PA. Dentinal hypersensitivity: a review. J Contemp Dent Pract. 2005; 6(2):107-17.

4. DW Barlett. Critical Review of Non-carious Cervical (Wear) Lesions and the Role of Abfraction, Erosion, and Abrasion J dent res 2006; 85:306-312,

5. Kazemi RB, Sen BH, Spangberg LSW. Permeability changes of dentine treated with titanium tetra fluoride. J Dent 1999; 27,531-538.

6. Gillam DG, Orchardson R. Advances in the treatment of root dentin sensitivity: mechanisms and treatment principles. Endod Topics 2006;13, 13-33

7. Azarpazhooh A, Limeback H. Clinical efficacy of casein derivatives: A systematic review of the literature Journal of the American Dental Association 2008; 139(7) 915-924.

8. Kumar VL, Itthagarun A, King NM. The effect of casein phosphopeptide-amorphous calcium phosphate on remineralization of artificial caries-like lesions: An in vitro study Australian Dental Journal 2008; 53(1) 34-40.

9. Reynolds EC. Anticariogenic complexes of amorphous calcium phosphate stabilized by casein phosphopeptides: a review. Spec Care Dentist. 1998 Jan-Feb; 18(1):8-16.

10. Reynolds EC. Casein phosphopeptide-amorphous calcium phosphate: The scientific evidence Advances in Dental Research 2009; 21(1): 25-29.

11. Reynolds EC, Cai F, Shen P, Walker GD. Retention in plaque and remineralization of enamel lesions by various forms of calcium in a mouthrinse or sugar-free chewing gum Journal of Dental Research 2003; 82(3):206-211.

12. Reynolds EC. Remineralization of enamel subsurface lesions by casein phosphopeptide-stabilized calcium phosphate solutions Journal of Dental Research 1997; 76(9)1587-1595.

13. Heymann HO, Bayne S. Current concepts in dentin bonding: focusing on dental adhesion factors. J Amer Dent Associatiion 1993; 124:27-37.

14. K.L. Van Landuyt, P.Kanumilli, J.De Munck, M. Peumans, P.Lambrechts, B. Van Meerbeek. Bond strength of a mild self-etch adhesive with and without prior acid-etching. Journal of Dentistry (2006) 34, 77-85

15. Brannstorm M. Smear layer: Pathological and treatment considerations. Operative dentistry: Supplement 3; 35-42.

16. Perdigao J. Dentin bonding as a function of dentin structure. Dent Clin North Am 2002; 46:277-301.

17. Bartold PM: Dentinal hypersensitivity: a review- Australian Dental Journal 2006; 51:(3):212-218

18. Hewlett ER: Etiology and Management of Whitening-induced Tooth hypersensitivity, CDA Journal 2007(35)7, 499-504.

19. Markowitz K, Pashley DH. Discovering new treatments for sensitive teeth: the long path from biology to therapy Journal of Oral Rehabilitation April 2008. 35(4): 300-315

20. Marchisio O, Esposito MR, Genovesi A. Salivary $\mathrm{pH}$ level and bacterial plaque evaluation in orthodontic patients treated with recaldent products. International Journal of Dental Hygiene 2010; 8(3) 232-236.

21. Llena C, Forner L, Baca P. Anti-cariogenicity of casein phosphopeptide amorphous calcium phosphate: a review of the 
literature Journal of Contemporary Dental Practice 2009; 10(3):1-9.

22. ParadellaTC. Current adhesive systems in dentistry- what is being said and researched. Odontologia Clin Cientif 2007; 6(4): 293-98.

23. G -Bond, Advanced 7th generation single component adhesive. Available at www.gcamerica.com.

24. Marshall Jr GW, Marshall SJ, Kinney JH, Balooch M. The dentin substrate: structure and properties related to bonding. Journal of dentistry 1997; 25: 441-58.

25. Sattabanasuk V. The bond of resin to different dentin surface characteristics. Oper Dent 2004; 29-3: 333-341.

26. Mc Cabe J.F, Ruby S. Dentin bonding agents- characteristic bond strength as a function of dentin depth. J Dent 1992; 20: 225-230.
27. Ogata M, Harada N, Yamaguchi S. Effect of different abrasives on the dentin bond strengths of self etching adhesives. Oper Dent 2001; 4: 375-382.

28. Pashley DH, Carvalho RM. Dentin permeability and dentin adhesion. Journal of dentistry 1997; 25: 355-72.

29. Van Meerbeek B et al. Adhesion to enamel and dentin: Current status and future challenges. Oper Dent 2003; 28-3: 215-35.

30. Van Landuyt KL, Kanumilli P, De Munck J, Peumans M, Lambrechets P, Van Meerbeck B. Bond strength of a mild self etching adhesive with or without prior etching. Journal of Dentistry 2006; 34: 77-85. 